\title{
What Do Parents Have to Do with My Cognitive Reserve? Life Course Perspectives on Twelve-Year Cognitive Decline
}

\author{
Hector M. González ${ }^{a-c}$ Wassim Tarrafa Mary E. Bowen ${ }^{d}$ \\ Michelle D. Johnson-Jennings ${ }^{\mathrm{e}}$ Gwenith G. Fisher ${ }^{\mathrm{c}}$ \\ ${ }^{a}$ Institute of Gerontology, and ${ }^{b}$ Department of Family Medicine and Public Health Sciences, \\ Wayne State University, Detroit, Mich., 'Institute of Social Research, University of Michigan, Ann Arbor, Mich., \\ ${ }^{\mathrm{d}}$ Research Center of Excellence, Veterans Health Administration, Tampa, Fla., and ePharmacy College, \\ University of Minnesota, Duluth, Minn., USA
}

\section{Key Words}

Cognitive reserve $\cdot$ Older adults $\cdot$ Life course $\cdot$ Development

\begin{abstract}
Background/Aims: To examine the cognitive reserve hypothesis by comparing the contribution of early childhood and life course factors related to cognitive functioning in a nationally representative sample of older Americans. Methods: We examined a prospective, national probability cohort study (Health and Retirement Study; 1998-2010) of older adults ( $n=8,833$ ) in the contiguous 48 United States. The main cognitive functioning outcome was a 35-point composite of memory (recall), mental status, and working memory tests. The main predictors were childhood socioeconomic position (SEP) and health, and individual-level adult achievement and health. Results: Individual-level achievement indicators (i.e., education, income, and wealth) were positively and significantly associated with baseline cognitive function, while adult health was negatively associated with cognitive function. Controlling for individual-level adult achievement and other model covariates, childhood health presented a relatively small negative, but statistically significant association with initial cognitive function. Neither individual achievement nor childhood SEP was statistically linked to decline over time. Conclusions: Cognitive reserve purportedly acquired through learning and mental stimulation across the
\end{abstract}

life course was associated with higher initial global cognitive functioning over the 12-year period in this nationally representative study of older Americans. We found little supporting evidence that childhood economic conditions were negatively associated with cognitive function and change, particularly when individual-level achievement is considered.

Copyright $\odot 2013$ S. Karger AG, Basel

\section{Introduction}

In upcoming decades, Alzheimer's disease and other dementias are projected to climb the ranks of the leading causes of disability worldwide [1]. Intensive research efforts to develop agents to arrest the progression or find cures for dementia are ongoing; however, those rewards have remained elusive. Further, if new agents are found, the availability of any new therapies to persons outside of highly specialized tertiary care units in high-income countries is uncertain. Presently, identifying targets for intervention that are low cost and accessible to most people offers some promise to reduce the looming disease burden of dementia worldwide [1].

Several studies have reported that regular and complex mental stimulation (e.g. educational and occupational achievement) is associated with a lower risk of cognitive decline and dementia in later life $[2,3]$. Originally

\section{KARGER}

E-Mail karger@karger.com

www.karger.com/ned
(C) 2013 S. Karger AG, Basel

0251-5350/13/0412-0101\$38.00/0
Hector M. González

Institute of Gerontology, Wayne State University

87 East Ferry Street, 226 Knapp Building

Detroit, MI 48202 (USA)

E-Mail hmgonzalez@med.wayne.edu 
demonstrated in nonhuman models $[4,5]$, enriched and mentally stimulating environments were associated with increased neuronal development. In accordance with nonhuman studies, mental stimulation purportedly increased neuronal capacity and efficiency to withstand insults and forestall cognitive decline and dementing conations. This higher 'threshold' or capacity to endure neuronal insults has been referred to as the brain or cognitive reserve hypothesis [6]. Though findings from previous work are mixed [7], a lifetime of enhanced mental stimulation, often measured by educational and occupational achievement, may confer lowered risk for cognitive decline; however, they may also serve as proxies for other factors, such as genetics and better childhood nutrition. In a systematic review, Fratiglioni and Wang [3] argued for a life course approach to understanding important 'time periods' that may influence the development of chronic disorders with long latencies before onset, such as heart disease [8], disability [9], and dementia [3]. While there is some evidence that childhood cognition affects cognitive reserve independently of adult educational attainment and occupation [10], few studies have examined childhood socioeconomic position (SEP) and later cognitive functioning and these have largely been cross-sectional and focused on younger adults [11-13]. The longitudinal study that has evaluated childhood SEP in relation to cognitive decline in later life among Blacks in Chicago have shown small effects [14-16]. Indeed, while a life course approach may identify critical time periods associated with later cognitive decline, no studies have evaluated childhood SEP and adult achievement simultaneously. Furthermore, no studies, to our knowledge, have compared the relative contribution of time periods that could affect later cognitive development, including decline. The purpose of this study was to examine the relative contribution of childhood SEP and adult achievement to later-life cognitive function and change over 12 years of a nationally representative cohort of older adults.

\section{Methods}

\section{Data}

We analyzed data from the 1998 through 2010 biennial waves of the Health and Retirement Survey (HRS). The HRS is a longitudinal, nationally representative sample of noninstitutionalized adults aged 51 years and older (with cohorts born between 1900 and 1947) living in the contiguous United States. The HRS is an ongoing study that is conducted by the University of Michigan, Survey Research Center with support from the National Institute of Aging and the Social Security Administration. The HRS sample design was based on a four-stage area probability sample of US households with at least one noninstitutionalized individual aged 51 years or older (i.e., born in 1947 or earlier). More detailed descriptions of the HRS sampling frame have been previously published $[17,18]$. Original survey response rates ranged between 70 and $81 \%$ depending on age cohorts. The University of Michigan Health Sciences and Behavioral Sciences Institutional Review Board approved the HRS.

\section{Baseline Sample}

From the sample of eligible respondents $(n=21,384)$ interviewed at the 1998 baseline wave, we retained the subsample of interest that is comprised of respondents 65 years and older $(\mathrm{n}=$ 10,777 ). We excluded 1,272 proxy-interviewed respondents who, according to the HRS protocol, were not administered cognitive tests at baseline. Additionally, to avoid bias introduced by individuals possibly affected by brain diseases, we excluded the bottom $5 \%$ of cognitive test scorers $(n=672)$. Our available sample at baseline was $n=8,833$, which, when weighted, represented 27,816,179 adults aged 65 years and older in the USA in 1998.

\section{Attrition}

Sample attrition was primarily attributable to mortality with the per-wave death rates varying between $6.5 \%$ in 2000 and $9.8 \%$ in 2010. Cumulative attrition due to mortality between 1998 and 2010 was $50.7 \%$. Non-death-related nonresponse was small and stable across waves and ranged from a low of $4.2 \%$ in 2000 to a high of $5.3 \%$ in 2010 .

\section{Main Outcome Measure}

Cognitive functioning (range $0-35$ points) was the outcome variable of interest. Cognition was assessed in the HRS using an abbreviated version of the Telephone Interview of Cognitive Status (TICS) [19], which is a composite of cognitive tests used to evaluate memory, attention, concentration, orientation, and language [20]. This measure is reliable and valid and has been used in numerous previous studies [21, 22]. It is comprised of two main dimensions: episodic learning and memory and mental status [23]. Specifically, 20 of the 35 TICS total points involve list learning and memory (i.e., episodic learning and memory), whereas the remaining 15 points are related to mental status type questions (i.e., orientation) and working memory (i.e., serial 7's and backwards counting). Comprehensive additional test characteristic descriptions are available at the University of Michigan, Institute of Social Research website (http://hrsonline.isr.umich. edu/index.php? $p=$ userg). The TICS was administered to all HRS respondents (except proxy interviews) in English or Spanish by trained and supervised interviewers, including the subpopulation in this study aged 65 years and older, at each of the 7 biennial study waves examined (1998-2010).

\section{Primary Predictors}

Baseline self-reported childhood and adult SEP and health status were the primary predictors. Childhood SEP was measured by respondent reports of father's and mother's education $(0=$ missing, 1 = less than high school, 2 = high school or more), and selfreport of overall childhood socioeconomic status (i.e., 'pretty well off, 'average or varied', and 'poor'). Childhood health was measured using a 5 -category scale $(1=$ excellent to $5=$ poor $)$ treated as a continuous indicator. Adult individual achievement was as- 
sessed using 3 indicators measuring respondents' education (0-17 years or more), household income, and household wealth. Household income was measured for the previous full calendar year, and comprised of a sum across all sources of income (e.g. employment income, pensions, Social Security, stocks, and others). Missing data were imputed. Wealth was measured by the sum of valued assets (e.g. home). Income and wealth were provided by self-report by the household financial respondent (i.e., among households with both spouses interviewed in the study, the household financial information is only gathered from the one respondent who is most knowledgeable about the household's finances). The same values for income and wealth, respectively, are applied to both individuals in households in which both spouses participated in the HRS.

Both household income and wealth were log-transformed to normalize their distributions. To avoid generating missing values for zero or negative reports, both measures were censored at a USD 100 minimum with values below this threshold recoded as the minimum $[24,25]$. Adult health status was measured using two indicators. The first was a self-reported health status measure coded on the same scale as the childhood self-reported health status (i.e., 1 = excellent to 5 = poor). The second was a count of 8 self-reported health conditions including: high blood pressure, diabetes, cancer, lung problems, heart conditions, stroke, psychiatric problems, and arthritis. The other model covariates were demographic variables including: sex, race (non-Latino White, Black, Latino, and other), and age in years. To facilitate interpretation of the latent growth parameters, our income and wealth indicators were centered at their estimated sample mean, adult education was centered at 12 years, and age was centered at 65 years.

\section{Analytic Approach}

Univariate and cross-sectional analyses were done using the Stata 12.1 software (Stata, 12.1, College Station, Tex., USA, 2011) and latent growth curve (LGC) analyses were conducted in the Mplus 6.1 software [26]. Mplus has a flexible platform that allows modeling of both observed and latent constructs within the same framework and can accommodate a wide range of measurement types and statistical distributions. All statistical analyses performed accounted for the HRS survey design including sampling weights, clustering and stratification $[27,28]$. We configured the growth models as balanced on time (7 waves of interviews) with data missing at random [29]. Statistical analyses for LGC were conducted with multiple linear regressions, a full-information maximum likelihood estimation method with Huber-White covariance adjustment that produces robust estimates in the presence of nonnormality and nonindependence of observations [26, 30]. Full-information maximum likelihood allowed us to use all available data in the estimation of the latent growth models of interest [29], by assuming that the missing data are missing at random and that the hypothesized models are the same for complete and incomplete data subsets. To minimize the effect of missing data in the outcome variables, and overcome nonmissing at random data complications, the HRS staff imputed missingness when appropriate to yield a more complete data. Models fit to data from participants with complete observations in at least $3,4,5,6$, and all 7 waves produced trends similar to the ones reported in this study (diagram available on request) and provided substantive evidence to the robustness of our findings.

Life Course and Cognitive Reserve
Latent (SEM) formulations of growth curve models are similar to multilevel random coefficient models in that they provide information on intrasubject longitudinal profiles as well as intersubject (population) growth estimates [30,31]. The fixed-effects coefficients represent average growth estimates for the entire population while the random effects reflect individual variations in those population level coefficients [30,32]. Given the importance of time coding in the estimation and interpretation of growth curve models, we tested our unconditional growth curves using three parameterization scenarios including linear, quadratic and free form. Table 2 presents the generated fit indices and growth parameter estimates for each configuration. Following standard practice, we incrementally built and tested our LGC models [32]. Model 1 was a basic growth curve model, model 2 accounted for the demographic covariates, models 3 and 4 assessed the effects of childhood SEP and health factors, model 5 accounted for individual achievement, and finally model 6 was a comprehensive model accounting for the additional effects of adult health and including all of our predictors and covariates. Table 3 presents the parameter estimates and a comprehensive list of fit indices resulting from these models.

\section{Results}

Table 1 presents the sample characteristics at baseline. The mean age of the participants was 73.9 years. Women constituted about three fifths of the sample. Consistent with Census 2000 data, respondents over age 65 years were predominantly non-Latino Whites (87.6\%), with 4.1\% Latinos, and 6.7\% Blacks. About half of the respondents had about a high school education (mean $=11.9$ years). The average household income equaled USD 37,728 and the average household wealth was USD 331,740 . The average reported adult health status was 2.9 (range 1-5) with about $31 \%$ of participants reporting fair or poor health. The mean number of reported health conditions was 1.8 (range 0-8). Slightly higher than 1 in 7 respondents reported less than high school maternal $(71.7 \%)$ and paternal (71.1\%) education. Close to 12 and $15 \%$ of study participants did not report any maternal or paternal education information. A majority of respondents (60.8\%) reported an average or varying childhood socioeconomic status, while $33.3 \%$ reported living in poverty, and the remainder (6\%) indicated being financially 'well off' during childhood. The average reported childhood health was 1.8 (range 1-5), with close to three quarters of participants reporting very good or excellent health.

\section{Growth Models}

Our analysis of varying configurations of time coding did not show any substantively appreciable enhancement in model fit for the freely estimated or quadratic models 
Table 1. Demographic characteristics (weighted) of HRS respondents 65 years and older $(n=8,833)$ at the baseline wave (1998)

\begin{tabular}{|c|c|c|}
\hline & Percentage & SE \\
\hline \multicolumn{3}{|l|}{ Categorical indicators } \\
\hline \multicolumn{3}{|l|}{ Race/ethnicity } \\
\hline White & 87.6 & 1.0 \\
\hline Black & 6.7 & 0.6 \\
\hline Latino & 4.1 & 0.6 \\
\hline Other & 1.6 & 0.2 \\
\hline \multicolumn{3}{|l|}{ Sex } \\
\hline Male & 40.7 & 0.5 \\
\hline Female & 59.3 & 0.5 \\
\hline \multicolumn{3}{|l|}{ Childhood SEP } \\
\hline Pretty well & 5.9 & 0.3 \\
\hline About average & 60.8 & 0.8 \\
\hline Poor & 33.3 & 0.7 \\
\hline \multicolumn{3}{|l|}{ Mother education } \\
\hline Missing & 11.7 & 0.5 \\
\hline Less than high school & 71.7 & 0.6 \\
\hline High school or more & 16.6 & 0.6 \\
\hline \multicolumn{3}{|l|}{ Father education } \\
\hline Missing & 15.3 & 0.5 \\
\hline Less than high school & 71.1 & 0.7 \\
\hline \multirow[t]{2}{*}{ High school or more } & 13.6 & 0.5 \\
\hline & Mean & SE \\
\hline \multicolumn{3}{|l|}{ Continuous indicators } \\
\hline Age, years & 73.9 & 0.1 \\
\hline Total income, USD & 37,728 & 908 \\
\hline Total wealth, USD & 331,740 & 13,535 \\
\hline Education, years & 12.1 & 0.1 \\
\hline Number of conditions & 1.8 & 0.0 \\
\hline Adult health score & 2.9 & 0.0 \\
\hline Mother education, years & 8.7 & 0.0 \\
\hline Father education, years & 8.4 & 0.1 \\
\hline Childhood health score & 1.8 & 0.0 \\
\hline Number of waves & 4.9 & 0.0 \\
\hline
\end{tabular}

over the linear parameterization (table 2). Similarly, the estimated growth parameters and the derived time point mean estimates of cognitive decline produced largely similar average population growth curves. Model estimates under all 3 scenarios provided a favorable reflection of the sample estimates. Given the lack of substantial differentiation, and to facilitate interpretation of model covariates estimates and tests, we chose to pursue a linear parameterization of cognitive decline to examine the effects of our primary predictors and covariates [32].

Table 3 presents the estimates from the 6 tested LGC models and includes a detailed accounting of fit measures used to assess them. Model fit was progressively improved by our incremental accounting for the predictors and covariates of interest. Both growth factors (the intercept and the slope) in the unconditional model (model 1) were statistically significant. The mean initial status was 22.64 (range $0-35$ ) and the rate of decline in average cognitive scores was estimated at 0.84 points per wave.

The results under model 2 show the negative effects of age, and being African American on both the baseline status as well as cognitive performance over time. Both Latinos and respondents self-classifying as 'other' had lower initial cognitive scores, but did not differ in the slope of decline relative to Whites. Females had a higher initial cognitive status compared to males, but presented a steeper decline over time. Finally, an attrition effect, measured as number of waves of participation, was positively associated with both initial status and over time cognitive maintenance. Demographic factors explained $28 \%$ of the interindividual variance in initial cognitive status and $20 \%$ of the variance in the rate of change.

Adding our childhood SEP indicators (model 3) increased the explained intercept variance by $4 \%$, but had no explanatory effects on the slope variance. Higher childhood SEP, including higher self-reported SEP and paternal (both mother and father) education, was associated with better initial performance. However, none of the considered indicators presented a significant association with the estimated rate of change. Similar effects were evidenced for self-reported childhood health (model 4), with individuals reporting worse status presenting lower initial cognitive scores, but no effects on the rate of cognitive decline. Accounting for childhood health had minimal impact on explaining either of the model random coefficients.

Models 5 and 6 incrementally incorporated our adult achievement and health status predictors. As with our childhood SEP indicators, all 3 adult achievement measures (i.e., income, wealth, and education) were positively and statistically significantly associated with initial cognitive status (model 5). However, neither presented a significant effect on the rate of change. Accounting for adult achievement significantly attenuated the statistical effects of the considered childhood SEP and health status measures. Furthermore, including adult achievement in the model explained an additional $18 \%$ of the interindividual variance in initial cognition. No added explanatory effects were evidenced for the rate of change variance.

Model 6 considered the added effects of adult health status. Lower assessment of health was associated with decreased cognitive performance at baseline, but had no 
Fig. 1. Predicted global cognitive scores trajectories among US community-dwelling adults (65 years or older). Results are based on longitudinal growth modeling of data from the HRS (19982010). (1) = Average age, White female, average adult achievement, high child SEP, high adult and child health; (2) = average age, White female, average adult achievement, low child SEP, high adult and low child health; (3) = average age, White female, average adult achievement, high child SEP, low adult and high child health; (4) = average age, Black female, average adult achievement, high child SEP, high adult and child health; (5) = average age, Black female, average adult achievement, low child SEP, high adult and low child health; (6) = average age, Black female, average adult achievement, high child SEP, low adult and high child health.

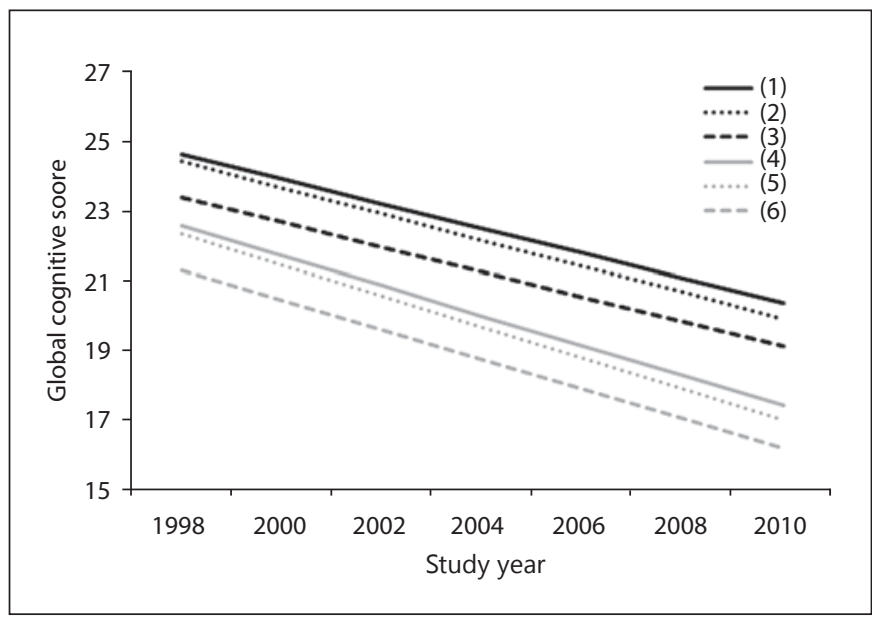

Table 2. Unconditional LGC parameter estimates for global cognitive score changes using linear, free, and quadratic time parameterizations and model fit indices

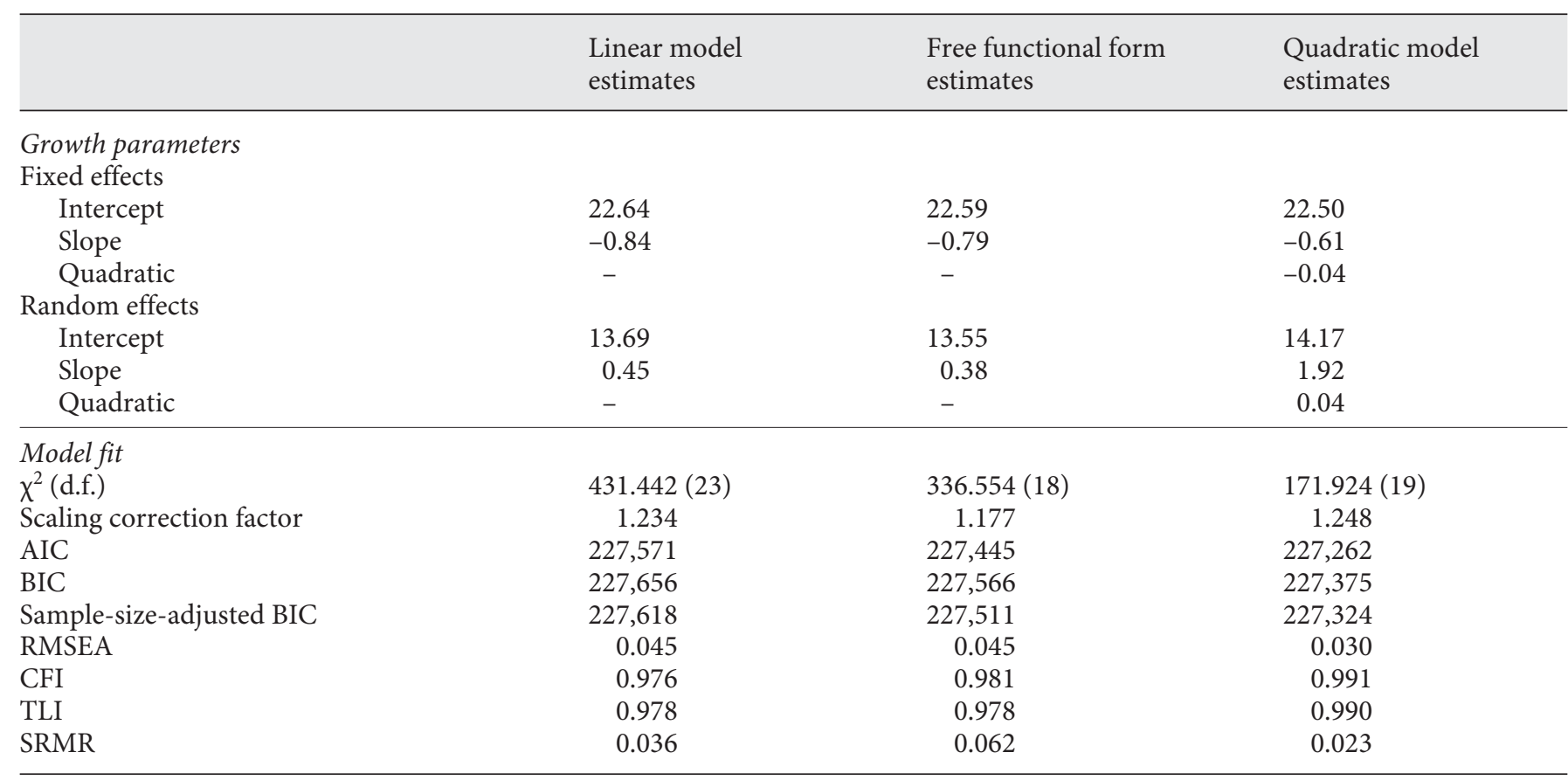

Weighted estimates are for US community-dwelling adults (65 years or older) from the HRS (1998-2010). AIC = Akaike information criteria; $\mathrm{BIC}=$ Bayesian information criteria; RMSEA = root mean square error of approximation; CFI = comparative fit index; TLI = Tucker Lewis index; SRMR = standardized root mean square residual.

statistical effects on the rate of change. A higher count of medical conditions was not associated with initial cognitive status or cognitive change over time. Incorporating adult health further attenuated the effects of childhood health on the baseline cognitive status. Including adult health had a marginal added impact (2\%) on explained intercept variance and no effect on the variance in the rate of change. Figure 1 includes a graphical representation of several covariate effects on cognitive performance. 
Table 3. LGC model estimates ( $\beta$ with SE in parentheses) of global cognitive score changes among US community-dwelling adults (65 years or older) from the HRS (1998-2010)

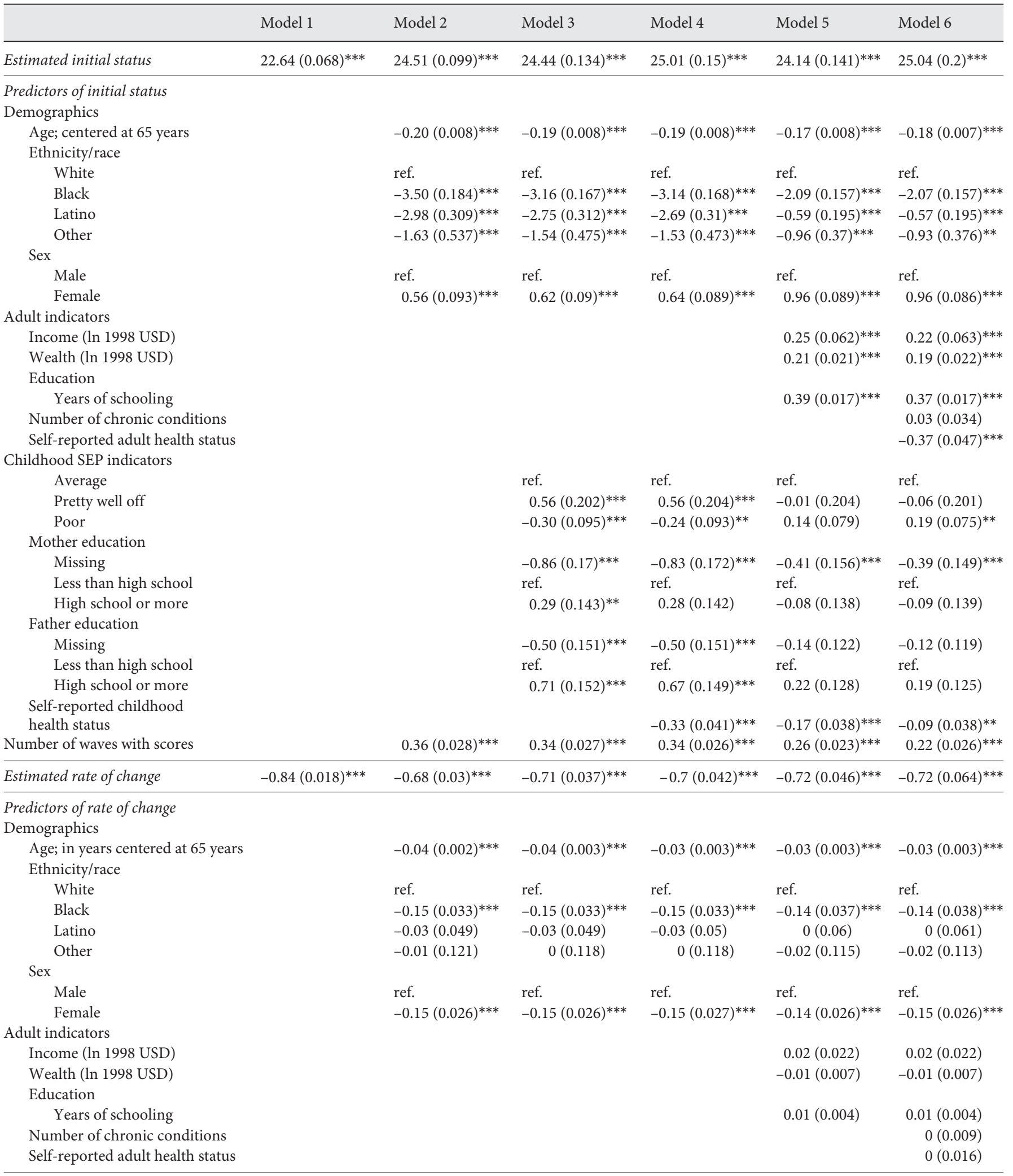


Table 3 (continued)

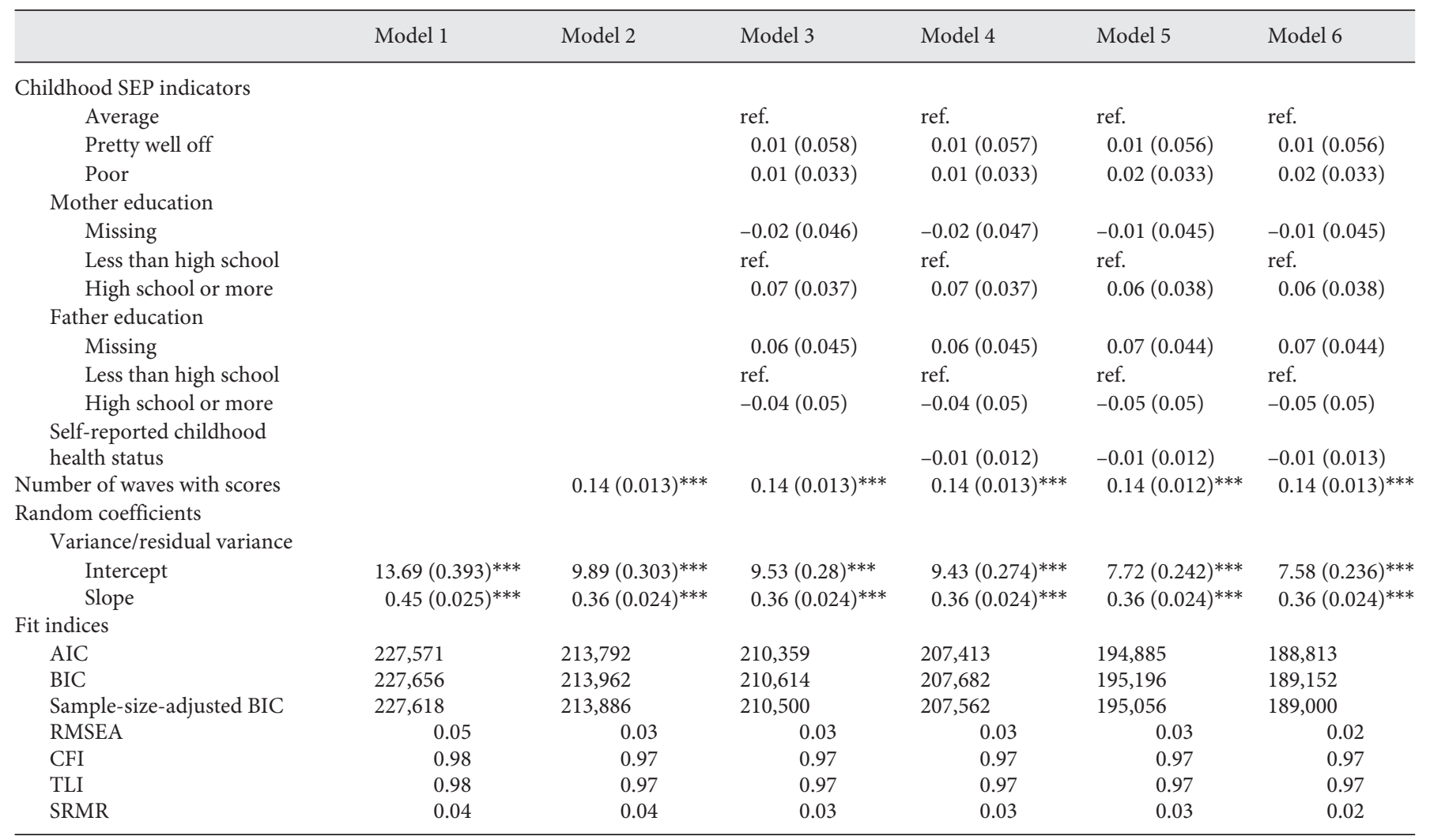

Model 1 is an unconditional growth model; model 2 accounts for respondents' demographic characteristics and number of waves of participation; model 3 accounts for demographic characteristics and childhood socioeconomic indicators; model 4 accounts for demographic characteristics and childhood socioeconomic and health indicators; model 5 accounts for demographic characteristics, childhood socioeconomic and health indicators, and adult socioeconomic achievement; model 6 is a comprehensive model that additionally accounts for adult health indicators. AIC $=$ Akaike information criteria; $\mathrm{BIC}=$ Bayesian information criteria; $\mathrm{RMSEA}=$ root mean square error of approximation; CFI = comparative fit index; TLI = Tucker Lewis index; SRMR = standardized root mean square residual. ${ }^{* * *} \mathrm{p}<0.01$; $^{* *} \mathrm{p}<$ 0.05 .

\section{Discussion}

We found that compared to childhood socioeconomic conditions, adult achievement largely explained global cognitive functioning in this nationally representative and longitudinal study of older adults. This may be because childhood conditions 'set the stage' for educational and subsequent occupational opportunities across the life course. Our study results support the cognitive reserve hypothesis assertion that ongoing adult mental stimulation attained through individual-level achievement (e.g. educational and wealth) serves to create an initial, protective buffer for cognitive function in older age. Secondly, we found limited support for the assertion that higher childhood SEP was associated with higher baseline cognitive function and no support for its association with slower decline $[11,12]$. Similarly to studies focusing on child- hood cognition [33], the findings from this study suggest that difficult childhood conditions may not be insurmountable and that interventions aimed at enhancing cognitive reserve have the potential to overcome their small adverse effects. Thus, opportunities for increased educational attainment, especially among children from low SEP families, and lifelong mental stimulation afford better adulthood occupational economic advantages and may lower health costs in later adulthood by forestalling and slowing later cognitive decline. Alzheimer's disease and related dementias will continue to impose greater disease burdens as the global older adult population expands [1]. Public health initiatives to maintain healthy brains should consider the long-term benefits of ensuring access to mentally stimulating opportunities (including educational and occupational) that afford these desirable benefits over the life course. 
Previous studies reported the independent relationships between childhood SEP, adult achievement, and cognitive functioning in later adulthood. We believe this is the first longitudinal study to evaluate the relative contributions of childhood and adult characteristics in relation to later cognitive function and decline. We found that respondents with higher childhood SEP had relatively lower baseline global cognitive function scores than those from modest childhood backgrounds. We believe that enhanced baseline cognitive performance of low childhood SEP respondents may reflect the economic position of most Americans during the pre-World War II, Depression era when most HRS respondents were reared. Compared to findings herein, higher childhood SEP has been associated with higher cognitive function among older adults - largely from Black and White urban neighborhoods; however, this association was largely mediated by educational achievement [14]. Similarly, cross-sectional studies of middle-aged Scandinavian men have reported that higher childhood SEP was related to higher cognitive test scores in adulthood $[11,12]$. In this study, we found marked differences in global cognitive function at baseline and later decline between Blacks and Whites. Of note was that the differences in cognitive scores for Blacks were highest regardless of childhood SEP or individual-level achievement, which suggests that other unexplored factors may be contributing to these pronounced differences. Whether or not the different samples explained the studies' differences awaits additional research.

The large, longitudinal, and nationally representative data used is a major strength of this study. The use of LGC analyses is an innovative analytic approach to examining how multifactorial, theoretical constructs (e.g. childhood SEP) related to outcomes of interest within a complex survey design. While life course approaches are often discussed in research studies to understand a variety of health outcomes, including cognitive functioning and decline; to our knowledge, none have systematically compared important life epoch's influences on later life cognitive function $[34,35]$.

Important caveats merit attention in interpreting our study results. First, due to data limitations, we exclusively examined childhood socioeconomic factors. Other important factors, such as childhood anthropometry, biomarkers, and cognition may have improved the available 'signal' from childhood factors in relation to later cognitive functioning. It is possible that the use of other childhood factors would have affected group cognitive score differences. Secondly, while we evidenced an overall decline in cognitive functioning over the 10year study period, the changes in scores were on average of moderate size. Examining subgroups at risk for cognitive decline and impairment would likely yield more pronounced score changes over time. Additionally, important to note is that our results represent a population of over 30 million older Americans. We believe that our model framing reduces the inherent biasing effects of error on our estimates by capturing multiple dimensions of childhood SEP and individual achievement. However, it should be mentioned that we used retrospective self-reports of childhood SEP that may be sensitive to recall bias and period effects (e.g. the Great Depression), and could have influenced the estimated relationship between childhood SEP and cognitive scores. Secondly, the global cognitive functioning score measure used herein has been demonstrated as a useful screening test for dementia and cognitive impairment; however, dementia was not ascertained in our study. Furthermore, it is likely that neuropsychologically, this composite cognitive measure may be more sensitive to adult factors (e.g. wealth) and relatively insensitive to specific changes in cognition related to childhood SEP. In addition, other more granular cognitive measures of specific neuropsychological domains could yield differing associations with childhood and adult factors. To our knowledge, this is the first longitudinal, nationally representative study to examine and compare the relative contribution of different important life course epochs in relation to cognitive aging. Further work is needed to test the hypothesis that certain life course periods have different relationships with specific cognitive domains.

\section{Conclusion}

Cognitive reserve purportedly acquired through learning and mental stimulation across the life course was associated with higher baseline global cognitive functioning in this nationally representative, 12-year longitudinal study of older Americans. We found little supporting evidence that childhood SEPs were related to cognitive function decline, particularly when adult achievement is also considered. We found that cognitive reserve via adult individual-level achievement may reverse any adverse effects of childhood SEP. Accessible learning opportunities provide economic and occupational rewards and may also afford protective benefits to having and maintaining healthy brain functioning in older adulthood. 


\section{Acknowledgements}

Drs. González and Tarraf were supported by the National Institute of Mental Health (MH 67726 and MH 84994) and the National Heart Lung Blood Institute (HC 65233). Dr. Fisher was supported by the National Institute on Aging (AG 027010, AG007137, and AG009740). Dr. Bowen was supported by a Rehabilitation Research and Development Career Development Award from the
Department of Veterans Affairs (CDA E7503W). The authors' views are their own and do not necessarily represent the views of the NIH or the VA.

\section{Disclosure Statement}

None to report.

\section{References}

$>1$ Mathers C, Loncar D: Projections of global mortality and burden of disease from 2002 to 2030. PLoS Med 2006;3:e442.

$>2$ Mortimer JA, Graves AB: Education and other socioeconomic determinants of dementia and Alzheimer's disease. Neurology 1993; 43(suppl 4):S39-S44.

$\checkmark 3$ Fratiglioni L, Wang HX: Brain reserve hypothesis in dementia. J Alzheimers Dis 2007; $12: 11-22$.

4 Diamond MC: Enriching Heredity: The Impact of the Environment on the Anatomy of the Brain. New York, Free Press, 1988.

$\checkmark 5$ Mohammed AH, Zhu SW, Darmopil S, et al: Environmental enrichment and the brain. Prog Brain Res 2002;138:109-133.

6 Katzman R, Terry R, DeTeresa R, et al: Clinical, pathological, and neurochemical changes in dementia: a subgroup with preserved mental status and numerous neocortical plaques. Ann Neurol 1988;23:138-144.

7 Bowen ME: A prospective examination of the relationship between physical activity and dementia risk in later life. Am J Health Promot 2012;26:333-340.

$>8$ Bowen ME: Coronary heart disease from a life-course approach: findings from the health and retirement study, 1998-2004. J Aging Health 2010;22:219-241.

-9 Bowen ME, Gonzalez HM: Childhood socioeconomic position and disability in later life: results of the health and retirement study. Am J Public Health 2010;100(Suppl 1):S197-S203.

10 Richards M, Sacker A: Lifetime antecedents of cognitive reserve. J Clin Exp Neuropsychol 2003;25:614-624.

-11 Kaplan GA, Turrell G, Lynch JW, Everson SA, Helkala E-L, Salonen JT: Childhood socioeconomic position and cognitive function in adulthood. Int J Epidemiol 2001;30:256-263.

- 12 Turrell G, Lynch JW, Kaplan GA, et al: Socioeconomic position across the lifecourse and cognitive function late middle age. J Gerontol B Psychol Sci Soc Sci 2002;57:S43-S51.

13 Luo Y, Waite LJ: The impact of childhood and adult SES on physical, mental, and cognitive well-being in later life. J Gerontol B Psychol Sci Soc Sci 2005;60:S93-S101.
4 Everson-Rose SA, Mendes de Leon CF, Bienias JL, Wilson RS, Evans DA: Early life conditions and cognitive functioning in later life. Am J Epidemiol 2003;158:1083-1089.

15 Bezerra ABC, Coutinho ESF, Barca ML, Engedal K, Engelhardt E, Laks J: School attainment in childhood is an independent risk factor of dementia in late life: results from a Brazilian sample. Int Psychogeriatr 2012;24:55-61.

16 Jefferson AL, Gibbons LE, Rentz DM, et al: A life course model of cognitive activities, socioeconomic status, education, reading ability, and cognition. J Am Geriatr Soc 2011;59: 1403-1411.

17 Juster F, Suzman R: An overview of the Health and Retirement Study. J Hum Resour 1995; 30(suppl):S7-S56.

18 Heeringa SG, Connor J: Technical Description of the Health and Retirement Study Sample Design. HRS/AHEAD Documentation Report DR-002. Institute for Social Research, University of Michigan, 1995.

19 Brandt J, Spencer M, Folstein M: The telephone interview for cognitive status. Neuropsychiatry Neuropsychol Behav Neurol 1988; 1:111-117.

20 Herzog A, Wallace R: Measures of cognitive functioning in the AHEAD Study. J Gerontol B Psychol Sci Soc Sci 1997;52(Spec No):37-48.

21 Ofstedal MB, Fisher GG, Herzog AR: Documentation of Cognitive Functioning Measures in the Health and Retirement Study. Ann Arbor, University of Michigan, 2005.

$\checkmark 22$ Rodgers WL, Ofstedal MB, Herzog AR: Trends in scores on tests of cognitive ability in the elderly US population, 1993-2000. J Gerontol B Psychol Sci Soc Sci 2003;58:S338S346.

23 McArdle J, Fisher G, Kadlec K: Latent variable analyses of age trends of cognition in the Health and Retirement Study, 1992-2004. Psychol Aging 2007;22:525-545.
24 Banthin JS: Changes in financial burdens for health care: national estimates for the population younger than 65 years, 1996 to 2003. JAMA 2006;296:2712-2719.

25 Gellad WF, Donohue JM, Zhao X, Zhang Y, Banthin JS: The financial burden from prescription drugs has declined recently for the nonelderly, although it is still high for many. Health Affairs 2012;31:408-416.

26 Muthén LK, Muthén BO: Mplus User's Guide, ed 6. Los Angeles, Muthén and Muthén, 1998-2010.

27 Satorra A: Complex sample data in structural equation modeling. Sociol Methodol 1995;25: 267.

28 Asparouhov T: Sampling weights in latent variable modeling. Struct Equ Modeling 2005; 12:411-434.

$29 \mathrm{Wu}$ W: Evaluating model fit for growth curve models: integration of fit indices from SEM and MLM frameworks. Psychol Methods 2009;14:183-201.

30 Bollen KA, Curran PJ: Latent Curve Models: A Structural Equation Perspective. Hoboken, Wiley-Interscience, 2006.

31 Bryk AS, Raudenbush SW: Hierarchical Linear Models: Applications and Data Analysis Methods. Newbury Park, Sage Publications, 1992.

32 Singer JD, Willett JB: Applied Longitudinal Data Analysis: Modeling Change and Event Occurrence. New York, Oxford University Press, 2003.

33 Tucker-Drob EM, Johnson KE, Jones RN: The cognitive reserve hypothesis: a longitudinal examination of age-associated declines in reasoning and processing speed. Dev Psychol 2009;45:431-446.

34 Bowen ME, González HM: Childhood Socioeconomic Position and Disability in Later Life: Results of the Health and Retirement Study. Am J Public Health 2010;100(suppl 1):S197-S203.

35 Lynch JW, Kaplan GA, Shema SJ: Cumulative impact of sustained economic hardship on physical, cognitive, psychological, and social functioning. N Engl J Med 1997;337:18891895. 